\title{
Autoatención y automedicación: reflexiones y retos desde la ontología del ser social
}

\section{Self-care and Self-medication: Reflections and Challenges from the Ontology of a Social Being}

\author{
Jorge Molina-Aguilar \\ Universidad Don Bosco, San Salvador, El Salvador y \\ Universidad Centroamericana José Simeón Cañas, San \\ Salvador, El Salvador
}

\begin{abstract}
Resumen
La autoatención, aunque la mayor parte del tiempo se considera como un problema de salud pública, también puede ser considerada un acto de empoderamiento y contrahegemonía; sobretodo, si se toma en consideración la autonomía de los individuos para aliviar y comprender una enfermedad o padecimiento. En el contexto institucional de la atención de la salud, la autoatención se convierte en un espacio donde conviven diversos tipos de instituciones, saberes y representaciones. Por lo anterior, los actores sociales que practican autoatención resignifican y descentralizan la práctica médica y el conocimiento científico. Una parte de esas prácticas es la automedicación. El objetivo de este artículo es explicar cómo la autoatención y la automedicación son parte de la vida cotidiana de los individuos, y cómo esta práctica no se limita a un ámbito médico; en cambio, propone ubicar la práctica de autoatención como parte de una discusión más amplia que tiene base en la ontología del ser social expresada en categorías como el hogar y lo cotidiano. Se plantean retos que requieren pensar en la autoatención y la automedicación desde un pluralismo disciplinar que va desde la antropología y la sociología médica, hasta la economía de la salud, la psicología y la farmacología. Se reflexiona en el hogar como primer espacio real de atención, donde se expresan prácticas laicas y tácticas microsubersivas; el cotidiano como un reflejo de aspectos históricos, sociales, coyunturales, políticos, ambientales, entre otros. Se plantea un modelo atención más allá del consultorio, lo vestal o privado, trasciende la idea del antiguo consultorio.
\end{abstract}

Palabras clave: autoatención, automedicación, cotidiano, hogar, híbridos, social

Jorge Molina-Aguilar, Universidad José Simeón Cañas, El Salvador.

La correspondencia en relación con este artículo se dirige a Jorge Molina-Aguilar, Universidad Don Bosco, San Salvador, El Salvador y Universidad Centroamericana José Siméon Cañas, San Salvador, El Salvador. Correo electrónico: 00503603@uca.edu.sv y jorge.molinaaguilar@protonmail.com 


\begin{abstract}
Self-care, although most of the time is seen as a public health problem, may also be considered an act of empowerment and counter-hegemony if it takes into consideration the autonomy of social groups to self-understand and self-alleviate a disease or illness. When it comes to the institutional health care context, self-care becomes a space where various types of institutions, knowledge, and representations coexist. Due to the foregoing, social actors practicing self-care re-signify and decentralize medical practice and scientific knowledge, by means of their practice of self-medication. The objective of this article is to explain how self-care and self-medication are part of an individual's everyday life, and how this practice is not limited to the medical realm; instead, it proposes to locate self-care practices as part of larger discussion about the ontology of a social being, expressed in categories such as the home and the everyday. But doing that poses a series of challenges that require thinking about self-care and self-medication from a perspective of disciplinary pluralism, ranging from anthropology and medical sociology to health economics, psychology and pharmacology. Home is reflected as the first real space of attention, where secular practices and micro-subversive tactics are expressed; also, everyday life as a reflection of historical, social, political and environmental aspects, among others. A care model is proposed beyond the office and the private, transcending the classical idea of a doctor's office.
\end{abstract}

Keywords: Self-care, self-medication, Everyday Life, Home, Hybrids, Social

A pesar de encontrarnos actualmente en un contexto de pandemia, el mundo ha vivido una serie de enfermedades que también demandó de medidas extraordinarias y, claro, un análisis que, desde sus inicios, reclamó un pluralismo disciplinar. Podemos situar algunos estudios SARS en Canadá y Taiwán en el 2004; los estudios sobre H1N1 en Australia en el 2013; y, no menos importante, el caso del ébola en Sierra Leona, Liberia y Senegal en el 2018 (Brooks et al., 2020).

Fue la Comisión Municipal de Salud de Wuhan, China, la que notificó públicamente un "conglomerado de casos de neumonía", y el 30 de enero de 2020 se estableció como causa el nuevo coronavirus. El 11 de marzo de 2020, la Organización Mundial de la Salud anunció oficialmente la pandemia. La misma institución planteó que no es solo una emergencia de tipo sanitaria, sino también social (Organización Mundial de la Salud [OMS], 2020).

El Salvador, con la meta de ralentizar la propagación del virus, adoptó al igual que una serie de países alrededor del mundo, desde el 22 de marzo de 2020, una cuarentena domiciliar y estado de excepción. Inicialmente, la cuarentena domiciliar fue decretada por un período de 15 días, los cuales se fueron extendiendo hasta terminar por ser una de las cuarentenas más largas del mundo. Resultó en 85 días. Se acompañó de un constante conflicto entre los poderes del Estado. Dicha situación conllevó a serias consecuencias y estragos a nivel económico y social, al igual que altos costos en la salud mental de la población salvadoreña (Molina \& López, 2020).

Un ejemplo fue el estudio de personas diagnosticadas con Lupus Eritematoso Sistémico (LES) y su vivencia en El Salvador durante el período de cuarentena. En dicho estudio, se evidenció cómo algunos pacientes deciden autoatenderse y asumir, desde el hogar, la automedicación con el objetivo de paliar las dificultades para conciliar el sueño (Molina, 2020).

Esta enfermedad, aparte de su compleja ambigüedad diagnóstica y pronóstica, constituye, en El Salvador, la segunda enfermedad reumática de mayor consulta después de la artritis reumatoide 
(Ministerio de Salud y Bienestar Social, 2019); aún así, las personas que padecen la enfermedad de LES, fueron, durante el período de cuarentena y estado de excepción en El Salvador, una de las poblaciones más afectadas en toda la región debido a que la coyuntura no solo restringió la consulta y el tratamiento médico, sino también la hidroxicloroquina, que es el medicamento especial en el tratamiento de LES. Así, se refuerza la cultura de automedicación en dicha población (Chicas, 2020; Molina, 2020). Inclusive, fue por movimientos sociales como FundaLupus que muchos pacientes buscaron la consecución del medicamento con medios alternos (Molina, 2020).

Con el Lupus, se evidenció como algunas formas (o manejos) en el abordaje tanto de "salud", "enfermedad", y "medicamento" no siempre armonizan con los modelos médicos tradicionales. Quienes exploran estos conceptos hacen una clara distinción teórica entre sistema social de salud y sistema cultural de salud (Castro \& Farmer, 2003; Farmer, 2000).

Desde la dimensión cultural, en algunas ocasiones - y como se desarrolla posteriormente- el primer espacio de atención es el hogar, donde se presenta cotidianamente una serie de decisiones importantes en torno a la salud y la enfermedad, a veces, al promover el consumo de medicamentos sin necesidad de tener previamente una consulta médica o una prescripción.

A fin de cuentas, el consumo de medicamentos (con o sin prescripción) implica una decisión, un comportamiento y, a la base de ellos, una valoración y una serie de motivos, que, de forma yuxtapuesta a factores de tipo histórico, social y cultural, tienen el potencial de culminar en una autoatención y muchas veces en la automedicación; o bien, en individuos que se abstienen de ingerir medicamentos o adherirse a un tratamiento específico, aún cuando se presenta una recomendación formal de parte del personal sanitario.

Este artículo propone un análisis sobre lo cotidiano, y sobre el hogar, a la base de la filosofía política de la vida cotidiana. Plantea un giro ontológico, puntualmente, desde la ontología del ser social, aborda al individuo como un ser social, un ser que asimila y, a veces, rechaza el conocimiento médico, un ser que no necesariamente se adhiere de manera lineal a un tratamiento. Por el contrario, es un ser que descentraliza y mezcla esa información médica con sus propias creencias, con sus valoraciones, con sus necesidades, y esto se da en su cotidiano, en su espacio doméstico, en su hogar, ambos (espacio y hogar) tradicionalmente están fuera del foco de investigación en profesionales en salud.

Este artículo es un esfuerzo por mostrar la relevancia que el hogar y lo cotidiano adquieren en las reflexiones sobre autoatención y automedicación. El texto pretende trascender un análisis coyuntural (pandemia) y, más bien, evidencia la presencia mínima de estudios en materia de farmacovigilancia desde un pluralismo disciplinar, desde las ciencias sociales y de la salud, pues estos han sido exclusivos a los campos de conocimiento farmacéutico y médico.

Tradicionalmente, la farmacoviligancia es un área de estudio de la farmacología; sin embargo, dentro de farmacología, también es posible investigar sobre "problemas vinculados a los medicamentos". Esto no es necesariamente exclusivo de la farmacología, pues temáticas como el abuso y mal uso de medicamentos pueden relacionarse con el estudio del comportamiento humano y, de hecho, son imprescindibles para mejorar la percepción de seguridad y riesgo (a los medicamentos) y fomentar la investigación en torno a su uso racional — no solo en profesionales del área farmacéutica y médica, sino todas aquellas profesiones afines a las ciencias de la salud (Organismo Salvadoreño de Reglamentación Técnica de El Salvador, 2016). 
Inicialmente, y de forma conceptual, es importante comprender las nociones de "híbridos" y "nuevos pacientes", desde la concepción teórica de Latour (1993), así como también la importancia del estudio sobre la filosofía política de la vida cotidiana desde la conceptualización de Heller $(1977,2004)$. Asimismo, se concibe al "paciente" más bien como un "actor social" al explorar específicamente sus motivos y expresiones en los espacios donde se desarrolla tanto la autoatención como la automedicación.

De este modo, se contempla el hogar no solo como vivienda o espacio físico, sino también como un primer espacio de autoatención y automedicación; es decir, un lugar donde se vinculan redes humanas y significativas, otros niveles de atención (familiares y amistades que influyen en el consumo de medicamentos) y también los patrones de automedicación (como resultado y como proceso sociohistórico), fruto del sistema económico capitalista y neoliberal — del cual no solo emerge una medicalización, mercantilización y patologización de la salud, sino también la ausencia de interculturalidad en temas de salud, y, en consecuencia, la infravaloración de los sistemas culturales de salud.

Por tanto, se desarrolló una revisión bibliográfica, la cual facilita "identificar huecos y oportunidades de investigación" así como también aporta un "marco teórico y metodológico que permite hacer aportaciones propias e incluso ser innovadores al afrontar nuevas investigaciones" (Codina, 2020, p. 141). La revisión bibliográfica puede servir como base fundamental en la producción académica; en este caso, en torno a la autoatención y la automedicación; este tipo de investigación aporta una "visión holística" al identificar alcances y limitaciones al igual que oportunidades en torno a la investigación. En esta ocasión, esta revisión se desarrolló principalmente a través de un pluralismo disciplinar desde las ciencias sociales (Codina, 2020).

Resultado de la investigación bibliográfica, se presenta una serie de abordajes conceptuales acerca de la automedicación y la autoatención desde diferentes paradigmas y, sobre todo, se enfatiza en conocer ambos conceptos desde una visión de proceso en contraste a una visión de resultado.

Para Codina (2020), las revisiones bibliográficas usan artículos de revistas científicas, obras de pensamiento, incluso ensayos, pues estos últimos de acuerdo al autor posibilitan conocer "los paradigmas dominantes en una disciplina" (Codina, 2020, p.143). En este caso, la base de la evidencia consistió en una revisión de artículos de revista, ensayos, libros, noticias publicadas en medios de comunicación, publicaciones en sitios especializados en salud, ponencias en congresos (sobre todo desde la antropología médica y de la salud), datos estadísticos de unidades de vigilancia sanitaria y reglamentos técnicos.

Estos documentos fueron obtenidos mediante bases de datos como EBSCOHost, AGORA, HINARI, MEDLINE Pubmed, Biblioteca Virtual en Salud, Google Scholar y bases propias del Ministerio de Salud y Bienestar Social de El Salvador, así como una serie de revistas especializadas entre las cuales es posible mencionar las siguientes: Wiley, Lancet, Canadian Science, Cuerpo Médico, Enfermería Neurológica, Antropología y Salud, Análisis Socioeconómico de El Salvador, BMC Proceedings, Revista Desacatos, Ciencia \& Saúde Coletiva, International Journal of Innovation Education and Research, Acta Sociológica, Papeles de población, Persona y Sociedad, Salud Uninorte, Sociology of Health \& Illness, Cuadernos de Antropología Social, Enfermería Global, Medisur, Medicent Electrón, Revista de Ciencias de la Salud, Ciencia e Investigación Médico Estudiantil Latinoamericana CIMEL, Archivos de Medicina, Acta Paulista de Enfermagen ACTA y la Revista Cuerpo Médico del HNAAA CM. De forma paralela, 
AutOATENCIÓN Y AUTOMEDICACIÓN

se realizó una revisión de documentos publicados en la División 28 Psychopharmacology and Substance Abuse de la American Psychological Association APA de la cual el autor es miembro.

Una vez identificados los documentos afines a las temáticas por explorar, se procedió a analizar y sintetizar las ideas principales y se encontraron contrastes en algunos casos, así como aproximaciones complementarias, alcances y limitaciones, abordajes y aproximaciones críticas, e incluso a descartar aquella documentación que no estaba enfocada en la temática. Por un lado, se profundizó en un contenido bibliográfico proveniente de diferentes disciplinas tales como la Antropología médica y la Antropología de la salud, la Sociología médica, la Farmacología, la Psicología y, por otro lado, desde especialidades tales como la economía de la salud y las teorías del consumo. Luego, se procedió a la construcción de un marco teórico donde se exponen los diferentes argumentos y paradigmas en torno a la autoatención, la automedicación y a la ontología del ser social.

Esta revisión consistió en el desarrollo de un marco teórico, el cual, de acuerdo con Codina (2020), se expresa generalmente como "síntesis narrativa", donde se analizan los espacios de consulta y la noción de "híbridos rebeldes" para dar pauta a reflexionar el fenómeno desde la ontología del ser social. Primero, se exponen las limitaciones que el modelo médico presenta en su abordaje de la automedicación y la autoatención y segundo, las categorías de cotidianidad y hogar desde la ontología del ser social.

Finalmente, producto de la revisión bibliográfica, se plantea una serie de reflexiones donde se exploran los alcances y las limitaciones de las teorías presentadas, así como los principales retos que surgen de pensar en la temática desde la ontología del ser social, principalmente, desde las categorías de hogar y cotidianidad mediante un pluralismo disciplinar.

\section{Abordaje conceptual}

La automedicación posee una diversidad de conceptos y definiciones. En algunos casos, por ejemplo, es definida desde la ingestión y el consumo. Para los casos anteriores, la autoatención y la automedicación se abordan en sintonía con otros conceptos tales como el autocuidado y el autodiagnóstico. Martínez-Cevallos (2013) considera la automedicación como la adquisición y la obtención de fármacos sin una prescripción médica por medio de círculos sociales cercanos. Desde esta conceptualización, la automedicación puede llevar no solo a prolongar o interrumpir tratamientos, sino también a incrementar o reducir la dosis al obtener resultados nocivos para la salud y el bienestar (Martínez-Cevallos, 2013).

Incluso, es conveniente tomar en consideración que las creencias acerca de los resultados pueden ser equivalentes o similares al tratamiento original. Dicha situación mantiene el comportamiento de automedicación.

\section{Paradigmas acerca de la automedicación}

Las definiciones acerca de la automedicación se enmarcan en distintos paradigmas. Algunos tienen como base la trasgresión y las consecuencias negativas. Aportando una visión acerca de la automedicación como una mala costumbre; otros, por el contrario, analizan la automedicación como proceso y como resultado, donde ambos (proceso y resultado) emergen de factores individuales, sociales y económicos (Cárdenas, 1998; Menéndez, 2003, 2018; Pardo, 1984; Sánchez \& Nava, 2012). 
Así, las definiciones pueden girar en torno a la automedicación como un hecho visto desde el consumo y al enfocar su atención a la acción de adquirir un medicamento sin consulta, sin supervisión y sin prescripción de parte de un profesional calificado (médico) (Lobo \& Carvalho, 2020; Pardo, 1984).

Calderón et al. (2020) expresan que la automedicación se puede dar en contexto de prescripción formal e implica cambios en la dosis prescrita o interrupciones en el tratamiento médico. También, en una segunda modalidad, el consumo de medicamentos se da sin prescripción, sin seguimiento o acompañamiento. En algunos países latinoamericanos, especificamente en las farmacias, existe la posibilidad de conseguir medicamentos sin fórmula médica; es decir, una disponibilidad del fármaco sin exigencia de una prescripción médica para su venta (Escobar-Salinas \& Ríos-González, 2017; Pari-Olarte et al., 2021).

A modo de ejemplo, un estudio muestra que el 35.17\% de individuos en Popaya, Colombia, adquiere sus medicamentos en la farmacia sin prescripción médica (Del Toro et al., 2020). Esto es similar a lo expuesto acerca de la automedicación en Brasil, en 1988, donde entre el 40\% y el 43\% de las personas adquirían el medicamento en las farmacias y, en complemento, el mismo estudio expone que, en Ecuador, el 51\% de las ventas de medicamentos se daba sin prescripción médica. Incluso, si estos tenían la posibilidad de tener efectos secundarios (González-López et al., 2012).

Otras investigaciones acerca de la automedicación en Latinoamérica demuestran cómo uno de los motivos principales es un "sistema de salud que permite la venta libre de medicamentos" (Oviedo et al., 2020, p.533). A modo de ejemplo, el mismo estudio expresa cómo el segundo motivo de la automedicación en Venezuela es el "fácil acceso y comodidad en un 50.49\%"; en este caso, de estudiantes universitarios (Oviedo et al., 2020, p. 538).

Por lo tanto, se evidencia no solo la facilidad de adquirir medicamentos, sino también de transmitir esta costumbre en Latinoamérica "de generación en generación” (Fernández et al., 2021, p.61). Incluso, en el caso de los psicofármacos, autores como Gómez et al. (2020) mencionan que a pesar de necesitar controles médicos obligados, las personas se valen de otras vías alternas para obtener este tipo de medicamentos, los cuales deberían tener prescripción.

Aparte del acto de consumo (con o sin prescripción), otras definiciones analizan la automedicación a partir de sus procesos (evidentes y subyacentes). Se consideran las dimensiones interculturales, que bien pueden expresarse en recomendaciones del botánico, del farmaceuta, del curandero, de los familiares o las amistades, entre otros; estas definiciones, también, consideran la forma en que estructuras macroeconómicas, contexto social, político, histórico y coyuntural están vinculadas a la automedicación (Cárdenas, 1998; Menéndez, 2003, 2018; Pardo, 1984).

\section{Proceso de la automedicación}

Sánchez y Nava (2012), previo a definir la automedicación, plantean el autodiagnóstico. Se entiende como un reconocimiento que los sujetos desarrollan acerca de la enfermedad o el padecimiento. Para estos autores, la automedicación se define como la ingestión de medicamentos de venta libre por iniciativa de los sujetos. Consiste en una práctica que forma parte del autocuidado de la salud entre "equipo médico" y "paramédico" (Sánchez \& Nava, 2012). 
AutoATENCIÓN Y AutomedicACIÓN

Cárdenas (1998) abona a esta noción (desde el autocuidado) y menciona que, en los años 1980, los programas sanitarios de autocuidado fomentaban la participación comunitaria. Pardo (1984) entiende el autocuidado como "lo que la gente puede hacer por sí misma para establecer y mantener la salud, prevenir y tratar las enfermedades" (p. 192). Estas acciones comprenden aspectos que oscilan entre la higiene, los estilos, los hábitos de vida, los factores socioeconómicos y, claro, la automedicación (Pardo, 1984).

Como se explicó antes, el concepto de automedicación también puede ser comprendido desde la ausencia de participación (médica y farmacológica). En estos casos, la automedicación es definida como el uso de medicamentos sin ninguna intervención médica. Refleja no solo la ausencia de un diagnóstico, sino también de un tratamiento (Fajardo-Zapata et al., 2013).

Pero, este tipo de (auto)consumo de medicamentos depende de muchos factores, los cuales, a pesar de su diversidad, son resultado de un sistema deficiente que se evidencia en las dificultades en torno a la disponibilidad y el acceso a los fármacos, la falta de comprensión acerca de los métodos de uso, el empaque, la disponibilidad de tiempo para buscar el medicamento, los factores socioeconómicos, el tipo de atención de parte del personal sanitario, la falta de credibilidad en el equipo médico sanitario y administrativo en las áreas de salud y una serie de prejuicios y comportamientos que las personas desarrollan ante la enfermedad, el equipo médico y los medicamentos (Fajardo-Zapata et al., 2013).

Esta forma de explicar el fenómeno plantea que el padecimiento y el medicamento se mezclan a una falta de credibilidad (en el medicamento) y una búsqueda de otras fuentes de información, como el internet, por ejemplo (Fajardo-Zapata et al., 2013). De este modo, quienes padecen la enfermedad descentralizan la información mediante diferentes modalidades. Estas fuentes de información pueden ser, inclusive, personas cercanas en su comunidad.

Pardo (1984) analiza el fenómeno de los prejuicios y significados desde otras aristas que, de forma sistémica, pueden contribuir a profundizar en la comprensión de la automedicación. Esta puede ser comprendida no solo como un resultado, sino también como proceso que, a su vez, es resultado y forma parte de una estructura mayor que se inserta en la población. Se articula con las relaciones históricas y económicas; es decir, se enmarca en una totalidad social.

Un ejemplo son los estudios de Rajan (2017) acerca de cómo la industria farmacéutica forma parte del mercado y se enmarca en la economía política al otorgarle un funcionamiento clave como una industria farmacéutica multinacional. Para el mismo autor, el capital se apropia de la salud y la transforma en un estado de bienestar. Esto se materializa en una categoría "abstracta" que está sujeta a los intereses del capital dominante e incursiona en su relación con la democracia (Rajan, 2017). Un ejemplo de esto se puede evidenciar en cómo muchas vacunas son negocios directos entre las compañías farmacéuticas y los gobiernos locales.

Según el European Environmental Bureau (2018), en torno a la industria farmacéutica, esta ha tenido diversos escándalos en cuanto a temáticas medio ambientales; a modo de ejemplo, el caso de la producción de antibióticos donde una buena parte se realiza en China y en India, estos liberan componentes farmacéuticos activos en el medio ambiente. Dichos componentes contribuyen al desarrollo de la resistencia a los antimicrobianos, lo cual amenaza la salud y afecta el medio ambiente (European Environmental Bureau, 2018). 
Pardo (1984) menciona cómo lo antes mencionado se vincula a la cadena de producción (de medicamentos y tecnología biomédica) y, por tanto, su análisis debe ser contemplado desde las relaciones de producción hasta los procesos de salud y enfermedad, la morbimortalidad, el diagnóstico y la curación. En consecuencia, en todos estos procesos se encuentran presentes componentes ideológicos, históricos, sociales y culturales, y estos, a su vez, se encuentran imbricados en una estructura económica y social, la cual, muchas veces, fomenta relaciones de dependencia y de dominación como resultado de un modelo económico y social.

Un ejemplo de esto fue el caso de la restricción de la hidroxicloroquina a pacientes con lupus de hospitales públicos durante el inicio de la pandemia. Esta situación llevó a que buscaran soluciones alternas en vista que el medicamento no era posible de entrar en el sistema público y la adquisición privada tenían un alto costo (Molina, 2020).

De este modo, la automedicación no es solo una decisión aislada, automática y propia de un sujeto ante la enfermedad, sino que emerge como resultado de una vinculación de tipo sistémico-estructural entre lo antes mencionado. Así, la salud no solo deja de ser un derecho (aunque nunca lo fue, al menos, en la práctica), sino que se reafirma como un bien de consumo.

Acerca de las teorías de consumo aplicadas al campo de la automedicación, es posible encontrar abordajes que buscan explicar las motivaciones en torno a los consumidores de medicamentos; en algunos casos, da por resultado una búsqueda inmediata de salud y/o alivio de un padecimiento (Ansari, 2018; Lobo \& Woranovicz, 2020).

Algunos de los factores vinculados al proceso del (auto)consumo de medicamentos incluyen la disponibilidad, la apariencia, el análisis de las actitudes de los consumidores, el aumento o la baja en el gasto familiar en temáticas de salud, los esquemas de fidelización de las farmacias, los servicios de valor agregado, la expansión en las localidades urbanas y rurales, la incursión de algunos gobiernos en las ventas asociadas a la industria farmacéutica y los comerciantes mayoritarios y minoritarios que en muchos casos organizan cadenas de farmacias (Ansari, 2018; Hartney, 2021).

Lo anterior permite evidenciar que asumir la automedicación como un resultado, desde un abordaje conceptual simplista y focalizado en lo individual (y en sus patrones), no solo invisibiliza los procesos antes mencionados, sino también impide reflexionar en la automedicación como una respuesta ante las necesidades no siempre cubiertas de la población en temas de salud. Será importante, de aquí en adelante, reflexionar en la automedicación no solo como una parte de la autoatención, sino también como una de tantas expresiones que surgen de sistemas económicos y sociales injustos.

\section{Espacios de consulta e híbridos rebeldes}

La noción de "híbridos" e "híbridos rebeldes": el primero se refiere al tipo de personas que mantienen la creencia en categorías puras, herméticas y, por tanto, cualquier mezcla o relación con otras categorías, es —ontológicamente - algo fuera de lugar, de este modo, categorías como "economía", "ciencia"

o "política" son vistas desde la mayor pureza posible. En contraste, el caso de los "híbridos rebeldes" es resultado de cómo una cultura cambia de forma cotidiana, y distinto a la pureza, las categorías si están mezcladas (Blok \& Jensen, 2011, como se cita en Dew et al., 2014; Latour, 1993). 
AutoATENCIÓN Y AutomedicACIÓN

Esta reflexión lleva a considerar que temáticas como "medicación" o "atención médica" no necesariamente son exclusivas de un campo de conocimiento, pues toda producción científica se enmarca en una sociedad, en un contexto sociocultural, en un marco histórico y, claro, en una coyuntura específica. Así, el estudio de la autoatención y la automedicación requiere un pluralismo disciplinar y metodológico, pues ambas se enmarcan en un mundo donde lo social, lo médico, lo biológico y lo farmacológico están entrelazados.

Para Dew et al. (2014), los espacios, como la clínica de atención médica, las farmacias, la botánica, la tienda de alimentos o la clínica de consulta de terapia alternativa, no son simplemente espacios físicos, sino también herramientas manipuladas por usuarios. De este modo, el concepto de hibridación proporciona un enfoque que rompe con las nociones y categorías ortodoxas. Estos "híbridos", desde la concepción de Latour y pensando en el usuario, no son consumidores pasivos, sino productores activos de prácticas de medicación 'híbridas' que trascienden un solo campo disciplinar como la medicina (Latour, 1995, como se cita en Dew et al., 2014).

\section{Espacio, interacción y concordancia}

Vale la pena mencionar que el espacio se entiende como una realidad intersubjetiva, la cual se construye en la interacción social (Augé, 1995). De esta forma, autores como Dew et al. (2014) expresan cómo los espacios no son simplemente marcos normativos, sino también herramientas que las personas manipulan, manejan o bien trabajan sobre ellas. Desde la Sociología, el hogar es un centro de prácticas, por lo que la medicación —o automedicación, en este caso concreto-, es un espacio de toma de decisiones importantes. Así, la prescripción, el tratamiento y el mismo diagnóstico se desarrollan más allá del territorio médico y farmacológico y, por tanto, estos espacios aparte de los médicos y farmacológicos, deben ser considerados para quienes se desempeñan en áreas afines a la salud.

Quienes residen en el hogar (tanto familiares como personas significativas), aparte de tomar decisiones, también ejercen acciones y tienen influencia en situaciones vinculadas a la prescripción y el diagnóstico. La información que es tradicionalmente constituida como un "hecho científico" propio del campo médico o farmacológico, es, de hecho, deconstruido y reconstruido en el hogar, basado en la experiencia, en saberes previos, en prejuicios y otros factores. Esta combinación no siempre tendrá un resultado armónico con las intenciones originales de quienes prescriben (Cárdenas, 1998; Dew et al., 2014).

Según Dew et al. (2014), desde la Antropología se reflexiona respecto a las prácticas de salud en el hogar, mientras que la Sociología reflexiona en cómo el conocimiento médico está actualmente desvinculado de espacios, como el hogar y la comunidad (Dew et al., 2014). Ambas disciplinas son imprescindibles en el estudio de los espacios donde se desarrollan las prácticas de salud al estudiar cómo estas (prácticas) pueden variar en diferentes culturas y la forma en que aspectos como el internet o las dificultades en el alcance de atención sanitaria afectan de forma directa o indirecta la concordancia (Dew et al., 2014).

La concordancia es comprendida como un acuerdo que emerge de la negociación entre quienes acceden a los servicios de salud (pacientes en este caso) y el equipo profesional; este último respeta o debería considerar el respeto en torno a las creencias de los pacientes acerca de si deben o no ingerir medicamentos (Dew et al., 2014). Sin embargo, reflexiones acerca de la interculturalidad llevaron a que en la década de los años 1990, los estudios de Mead y Bower (2000, como se cita en Dew et al., 2014) 
discutieran este concepto al abrir un debate que tomaría aún mayor fuerza que se explora a continuación y que cuestiona ¿cuál debería ser considerado el primer nivel de asistencia? (Cárdenas, 1998).

\section{Autoatención, autoprescripción y automedicación}

Existen diferentes formas de concebir tanto la autoatención como la automedicación. Como se dijo antes, su abordaje conceptual, depende en gran medida de los modelos y los paradigmas médicos, económicos y sociales a la base. Según la Organización Mundial de la Salud, más de la mitad de los medicamentos se prescriben y se distribuyen (venta y dispensa) de manera inapropiada y la mitad de quienes los consumen lo hacen de forma inadecuada (Fajardo-Zapata, et al., 2013). Esto lleva a pensar que, desde la salud pública, la automedicación puede ser un problema grave, porque conlleva a riesgos y efectos nocivos para quienes la practican. Inclusive, menciona "la resistencia a los antibióticos por parte de los gérmenes" (Fajardo-Zapata et al., 2013, p. 232).

De acuerdo con Pardo (1984), desde una mirada económica, específicamente desde la economía de la salud, las personas eligen aquellas alternativas que aportan mayor satisfacción o utilidad. Esta mirada asume que la utilidad que las personas experimentan dependerá del consumo de bienes y el resultado en su estado de salud. En este caso, la automedicación (resultado de la autoatención y la autoprescripción) es simplemente una alternativa más entre un abanico de posibilidades en el campo de la atención; esto se articula a factores personales o individuales que bien pueden ser culturales, demográficos y socioeconómicos y estos, a su vez, relacionarse con las peculiaridades de quien provee el servicio médico sanitario (Pardo, 1984). La expectativa humana de resolver de forma inmediata los problemas médicos y propios del campo de la salud, facilita y fortalece las prácticas de automedicación y autoatención.

\section{Modelo médico, automedicación y autoatención}

Mucho del conocimiento sobre la autoatención y la automedicación emerge del modelo médico hegemónico. Cárdenas (1998) define este modelo desde el biologismo, el cual garantiza su categoría "científica" y no solo le diferencia de otros sistemas de salud, sino también le jerarquiza; es decir, le otorga hegemonía y le aporta una conexión lógica y estrecha con sectores afines al poder. En consecuencia, el modelo médico hegemónico $(\mathrm{MMH})$ se diferencia y se sitúa por encima de otros modelos explicativos, a veces, sin cuestionamiento alguno: las personas asumen que procesos de tipo psicológico, social o cultural se encuentran subyugados al modelo médico, vistos como meramente anecdóticos y, en algunas ocasiones complementarios, al otorgarles fácilmente una categoría de inferiores (Cárdenas, 1998; Menéndez, 2003, 2018).

En contraste, desde la Antropología, Cárdenas (1998) y Menéndez (2018) reflexionan en la falta de importancia que se da a la autoatención doméstica de la salud: ambos enfatizan en que este tipo de (auto)atención no ha sido lo suficientemente dimensionada. Inclusive, Menéndez (2003) expresa cómo la autoatención y la biomedicina mantienen una relación estrecha donde se evidencian las representaciones y los significados de los sujetos en torno a la salud, la enfermedad y los procesos de curación. Desde 1998, en el Congreso Chileno de Antropología se cuestiona la necesidad de incursionar en dos áreas importantes: primero, en las dinámicas psicológicas y sociales en torno a la autoatención y la automedicación y segundo, en aspectos socioculturales que pueden llegar a incidir en la toma de decisiones en torno a la autoatención. En ese mismo congreso, se expone cómo la demanda de consulta médica es baja 
AutoATENCIÓN Y AutomedicACIÓN

debido a la autoatención como un factor cotidiano al enfatizar en las medicinas tradicionales indígenas y populares (Cárdenas, 1998).

Menéndez (2018) problematiza aún más en el fenómeno al afirmar que la Antropología no solo debe incursionar en la temática de autoatención de parte de una intelectualidad indigenista; sino, más bien, pensar en una antropología de procesos, donde, de manera interactiva, los sujetos sean considerados los principales actores sociales en el proceso de autoatención al pensar en dinámicas subyacentes en torno a la salud, la enfermedad, la asistencia e inclusive, a la concepción de "paciente". Así, se abre la discusión no solo a los sectores indígenas, sino también a otros sectores donde se practica la autoatención y la automedicación - como en poblaciones campesinas o sectores socioeconómicos de escasos recursos-; es decir, no solo pensar en las incompatibilidades entre padecimientos y sanación como resultado de cosmovisiones diferentes (Menéndez, 2018).

Ambos autores orientan, de forma complementaria, el término "autoatención": para Cárdenas (1998), se concibe que la Antropología aporta conocimientos valiosos sobre el proceso salud-enfermedad y la atención y se dirige a conocer las estructuras de saberes previos a la base de estos procesos. Cárdenas (1998) invita a pensar en los padecimientos y la enfermedad no solo como ente nosológico o patológico, y al medicamento como una cura, sino también como proceso y resultado de una construcción entre significados colectivos, históricos y culturales.

De este modo, el dolor, la atención y el medicamento poseen un significado particular. Lleva a pensar también en las relaciones de hegemonía y subalternidad entre distintos sectores sociales. Estas representaciones y significados se convierten en prácticas sociales, en hechos sociales, ejecutados por sectores específicos de la sociedad, y nuevamente, afectan de manera significativa la vivencia (y concepción) de la enfermedad, del medicamento y de la atención, entre otros.

Menéndez (2018) expresa que la autoatención debe ser entendida como un conjunto de saberes, de representaciones y de prácticas sociales de las cuales, tanto sujetos como grupos, se valen para diagnosticar y comprender las formas de "controlar, aliviar, soportar, curar y solucionar o prevenir los procesos que afectan su salud en términos reales o imaginarios" — esto sin necesariamente tener una intervención profesional- (Menéndez, 2018, p. 106).

Al retomar las ideas de Menéndez (2018) y Cárdenas (1998) emerge como resultado de esta conceptualización (de la autoatención) una valoración de la autonomía, pues todo esto implica una decisión $\mathrm{y}$, muy fácilmente, una autoprescripción. Bien sea fruto de condiciones socioeconómicas o coyunturas específicas (ver caso del Lupus al inicio), o como una práctica constante y permanente en los sujetos, la autoprescripción, al igual que la automedicación, pueden concebirse como resultado o como proceso en la autoatención (Menéndez, 2018).

Campos-Navarro (2016) menciona que la autoatención fácilmente es confundida con la automedicación, sobretodo por la biomedicina. Esto lleva a pensar (de forma casi lineal) en la autoatención como una forma autónoma de utilizar medicamentos; pero, la automedicación es solo una parte de la autoatención y este reduccionismo y pensamiento lineal consecuencia es resultado del saber biomédico (Campos-Navarro, 2016). 
De este modo, resulta posible argumentar que la salud no es solo fruto de programas ejecutados por el sistema sanitario o por una red de profesionales, o incluso como lo expresa Campos-Navarro (2016), de otras fuerzas sociales que operan entre saberes culturales y formas de atención, como ONGs o fundaciones, por ejemplo. Es posible reflexionar en que la salud y las intervenciones en torno a ella, tampoco se desarrollan únicamente en espacios como el consultorio o las unidades autónomas y profesionales del sistema de salud; más bien, es importante pensar que el primer nivel real de atención en salud se desarrolla en el espacio doméstico, en el hogar, en la comunidad e implica la autoatención.

De hecho, estos espacios forman parte del ciclo salud y enfermedad, de la autoprescripción y de la automedicación. La manera en que estas formas de atención sean consideradas dependerá, en gran medida, tanto de la cultura, como de los modelos dominantes en salud. Esto implica, inclusive, reflexionar en la filosofía de la salud y la enfermedad bajo la cual dichos modelos han sido construidos.

\section{Más allá de la trasgresión}

En algunos casos, como lo plantea Cárdenas (1998), la autoatención y la automedicación serán consideradas una "trasgresión derivada de conductas individuales incorrectas" (Cárdenas, 1998, p. 734). Esto impide que la relación entre atención profesional y la autoatención sean procesos articulados y complementarios; es decir, entre el espacio médico y la vida cotidiana. Más bien, esta noción de trasgresión sirve como garantía para las ideas tradicionales, donde ambas son formas de atención incompatibles y contradictorias con los modelos dominantes (biomédicos); da por resultado una concepción permanente donde la autoatención (en los espacios antes mencionados) es algo negativo y escindido de la posibilidad de cura o bienestar (Menéndez, 2018).

Es importante reconocer que las poblaciones tienen una diversidad de prácticas en relación con la salud, las cuales son vistas desde diferentes paradigmas y disciplinas. Estas prácticas tienen a la base una cultura, un conocimiento y una experiencia previa, un significado y una representación en quienes buscan respuesta ante un malestar, un padecimiento, una enfermedad y también ante la búsqueda de la salud (Cárdenas, 1998).

La autoatención y, sobre todo, la atención doméstica en temas de salud no debe ser infravalorada o considerada de forma automática como una "trasgresión"; sino, más bien, como una categoría de investigación —o al menos como punto de partida. Esto es así porque la relación entre las distintas formas de atención, bien sean desde la autoatención o desde la atención profesional, tendrán siempre una vinculación estrecha y concebirle de forma inmediata como una "mala costumbre" o una trasgresión (Vega-Romero \& Vega-Romero, 2013) puede llevar fácilmente a reducir el análisis en los procesos que dieron por resultado la autoatención y la automedicación en un inicio al invisibilizar aspectos como la jerarquización y la medicalización de la salud, un sistema regulatorio débil y una serie de factores socioeconómicos y de acceso a la salud de parte de la población.

Si la enfermedad y los padecimientos son vistos exclusivamente desde la mirada del modelo médico tradicional, es posible encontrarse frente a dos fenómenos que Cárdenas (1998) menciona: la ahistoricidad y la asociabilidad. El primero asume que, tanto enfermedad como padecimiento, emergen y evolucionan de manera autónoma en relación con los determinantes locales y sociales. El segundo reflexiona en la incorporación de aspectos sociales, pero de forma anecdótica, ajenos a la investigación y 
AutoATENCIÓN Y AutomedicACIÓN

el análisis profundo de espacios domésticos como el hogar y la comunidad, así como también la cultura y su relación con la salud.

\section{Cotidiano y hogar}

Antes de explorar con mayor profundidad el hogar y lo cotidiano, podemos mencionar algunas ideas base desde los conceptos marxistas - Marxistische Konzepte - con especial atención en la ontología del ser social, la cual destaca lo cotidiano (Márkus, 1974; Marx, 2005; Marx \& Engels, 2007). Lo anterior facilita el entendimiento acerca de la vida cotidiana, no solo en individuos que padecen alguna enfermedad y viven la autoatención, sino también de miembros en el equipo médico sanitario y médico especialista. En ambos casos, la ontología del ser social toma en consideración el contexto social, cultural e histórico de las personas, así como la coyuntura en la cual se encuentra el sistema de salud y su relación con otros sistemas afines. Esto permite analizar las relaciones de tipo sistémico-estructural que se desarrollan en los distintos niveles.

Vale decir que este apartado no pretende una revisión exhaustiva e histórica acerca de la ontología del ser social, más bien, busca sentar las bases acerca del estudio de lo cotidiano y el hogar en el contexto de la autoatención y automedicación. A modo de propuesta, ambas categorías (hogar y cotidiano) permiten comprender la relación dialéctica que puede estar presente en los distintos niveles de la sociedad en relación con la autención y la automedicación — ambas son consideradas como un hecho social.

En este caso, la ontología del ser social es vista a la luz de las ideas Heller (2004), quien explora lo cotidiano y cómo se integran distintos niveles (del cotidiano) en la vida diaria de los individuos y se expresan en su día a día. Para Heller (2004), los individuos son seres sociales; se presentan como seres particulares y, a la vez, genéricos, producto y expresión de sus relaciones sociales; también, son herederos y protectores del desarrollo humano.

De esta forma, un individuo es un ser humano singular que tiene una actitud consciente $-\mathrm{y}$ autoconsciente-, en relación con su condición de generalidad y es capaz de conducir y orientar su vida de acuerdo con sus actitudes sensibles e intelectuales; por tanto, es el ser social consciente de sí mismo. En complemento, Márkus (1974) desarrolla la idea de un individuo que vincula el ser social a su proceso histórico de autocreación para una nueva sociabilidad (Márkus, 1974; Heller, 2004).

\section{Viraje ontológico}

Desde la ontología del ser social, las ideas, las invenciones, los ideales, los pensamientos y otros aspectos de naturaleza similar no son adquiridos de manera espontánea. Más bien, estos nacen, crecen, fructifican y también se intersubjetivan, resultado de la sumatoria de experiencias de vida y relaciones sociales a lo largo de la vida. Así, desde esta perspectiva, los seres humanos forman parte de un entramado social, cultural, político y económico, que, a su vez, confluye con aspectos personales como los valores, los conceptos, los juicios y prejuicios; que, al mismo tiempo, se integran a una realidad específica y en un momento histórico en el cual se sitúa el individuo (Heller, 2004; Márkus, 1974).

Es posible pensar al individuo como una síntesis, o más bien, como una integración de múltiples determinantes históricos y sociales que, de forma dinámica - y dialéctica—, se presentan a diario: en el hogar, en lo doméstico y estos determinantes están en y para la vida cotidiana (Heller, 2004). 
La autoatención y una parte de ella (la automedicación), por ejemplo, emergen como derivados que están unidos tanto a la vida material (económica) como a la producción de ideas (significados, representaciones). La autoatención es, pues, un producto social; emerge de una relación entre seres humanos, sistemas económicos y sociales, relaciones sociales e históricas, conceptos, prejuicios, entre otros; integrando así tanto el ser social — consciente de sí mismo - y el mundo a su alrededor expresado en distintas dimensiones, modalidades y niveles — sean estos de naturaleza económica, política o cultural - mediatizados por eventos que derivan de su propia experiencia (la de los seres sociales), de sus expectativas, de sus creencias y motivaciones (ideas base retomadas de Marx \& Engels, 2007).

En consecuencia, la autoatención y la automedicación pueden reflexionarse también desde la noción de libertad. Se entiende como un campo de acciones reales en las decisiones entre una serie de alternativas realizables (Lukács, como se cita en Heller, 1977, p. 10). Pero, estas acciones "individuales" o "personales" están mediadas por una estructura, un sistema social, un contexto histórico, una situación coyuntural, una estratificación social, modos de producción, entre otros (Heller, 1977).

\section{Cotidianidad e interacción}

Lo cotidiano es parte de la vida en todo ser humano, aunque cada uno es impactado en modalidades distintas por esa misma cotidianidad, la cual expresa las relaciones sistémico-estructurales y los niveles de una totalidad social. La autoatención, la decisión que implica el autodiagnóstico y la autoprescripción, la vivencia de automedicarse, se enmarcan en lo cotidiano. Así, para Heller (1977), lo cotidiano se entiende como todo aquello que se produce y reproduce dialécticamente, en un movimiento eterno, en un mundo donde hay objetivaciones; pero, lo cotidiano es también una yuxtaposición de los eventos antes mencionados; expresa una secuencia - aparentemente caótica- de hechos, acontecimientos, objetos, substancias (medicamentos, por ejemplo), fenómenos, rutinas, significados, prejuicios, relaciones sociales, entre otros.

La atención doméstica y el hogar son espacios que forman parte del cotidiano y, si bien, desde una mirada meramente biomédica. Pensar estos espacios como una primera forma de atención en salud implica una cantidad de información que en un inicio parece caótica; es en esos espacios, en esos contextos, donde los sujetos sociales deciden, actúan, organizan y reorganizan en torno a la salud, a la enfermedad o la prescripción y la medicación. A su vez, estas formas de acción y decisión emergen de una multiplicidad de relaciones y estratificaciones sociales, que son el resultado de un proceso histórico y social. En otras palabras, sus acciones, aunque parezcan caóticas, en realidad, son fruto de una integración compleja que se presenta día tras día en y para su cotidiano (Lukács, como se cita en Heller, 1977).

La forma en que se aborda la enfermedad, la curación, la salud y la autoatención está relacionada con un proceso de conciencia del ser social; en consecuencia, asimilar o negar todo aquello vinculado a la autoatención (y automedicación) está derivado no solo de quien padece la enfermedad, o quien diagnostica (especialistas), sino también de las condiciones sociohistóricas del lugar donde se vive, de las relaciones sociales con los líderes de hogar, de las personas significativas en su círculo inmediato, incluso, de la coyuntura actual de pandemia por COVID-19 y los contextos económicos, políticos, culturales y afines a los sistemas y estructuras de salud en su entorno. 
AutOATENCIÓN Y AUTOMEDICACIÓN

Por consiguiente, pensar el hogar y lo cotidiano como categorías de investigación y trabajo en torno a la autoatención y la automedicación, implica reconocer la importancia de la interacción. Esta se comprende de la siguiente manera: "La interacción social puede definirse en sentido estricto como aquella que se da exclusivamente en las situaciones sociales, es decir, en las que dos o más individuos se hallan en presencia de sus respuestas físicas respectivas" (Goffman, 1991, como se cita en Galindo, 2014, p.193).

\section{El hogar, espacio de creencias laicas}

Los hogares pueden ser vistos como un centro "híbrido" de práctica en tema de medicación y automedicación, como se mencionó antes. En la práctica, son un primer centro de atención en salud y este fenómeno de atención emerge de una interacción social, el cual, nuevamente, se vive en y para el cotidiano. En ellos se encuentran una amplia red de asesoramiento por parte de los miembros (del hogar) y esta no necesariamente tiene sintonía con las prescripciones o diagnósticos médicos, si es que los hay. Aparte de una red de asesoramiento, también hay presencia y disponibilidad de medicamentos (estos pueden ser resultado de una enfermedad previa, o de un botiquín, por ejemplo) (Dew et al., 2014).

Algunos factores de la automedicación incluyen la influencia del farmacéutico, los familiares y amigos, la percepción que se tiene sobre el problema de salud (como enfermedad menor, padecimiento, entre otros), conocimientos previos y prejuicios sobre el fármaco, dificultad de acceso y disponibilidad del medicamento, costo del fármaco, adquisición mediante canales informales (medicamento disponible en casa o por amistades), tiempo prolongado de espera en la asistencia médica, maltrato en el hospital o centro de atención y evitación de gastos de consulta médica (Martínez-Cevallos, 2013).

Desde la Sociología médica, se concibe que, si bien el diagnóstico y la prescripción son dominios exclusivos del campo médico, estas actividades están presentes en los hogares (Dew et al., 2014). De este modo, propietarios, jefes, líderes de hogar y quienes padecen la enfermedad pueden desviar o rechazar las prescripciones con base en su propia experiencia y convertirse en "expertos" mediante su propia formación y necesidad, así como también por medio de procesos de experimentación que les llevan a determinar aspectos como "la dosis adecuada", cuándo los medicamentos deben ser ingeridos y cuándo no o la sustitución de medicamentos por otras prácticas - como la dieta o el descanso, por ejemplo.

Desde la Antropología médica y cultural, y desde la Sociología médica, es común estudiar los tipos de hogar, pensar en aquellos espacios domésticos abastecidos de medicamentos, de purgas y remedios caseros o adquiridos en la farmacia o la botánica (Dew et al., 2014). Estos (hogares) se estudian mediante la observación y la experimentación, o bien, la etnografía de longue durée (Manilowski, 1922; Manilowski, como se cita en Holmes, 2016, p.61).

Los hogares son espacios de producción y reproducción de conocimiento, donde se rechazan o se aceptan conocimientos científicos, biomédicos o farmacológicos (Cárdenas, 1998; Dew et al., 2014). Los conocimientos del hogar pueden ser resultado de una tradición que, a su vez, emerge de varias generaciones, de saberes producidos en otros espacios significativos tales como el trabajo, la escuela, y aquellos miembros que forman el círculo más cercano; como también del internety de los medios de comunicación masivos y alternativos; pues, tanto líderes como quienes padecen o adolecen la enfermedad, realizan una búsqueda donde se presenta no solo su experiencia, sino también la consulta a los medios disponibles y sus redes de apoyo. 
En el hogar se encuentra lo que William y Popay llaman "creencias laicas": una serie de conocimientos que representan un desafío para los profesionales en salud, puesto que se encuentran fuera de los mundos científicos. Inclusive, la información que proviene de dichos espacios puede resultar desorganizada y descentralizada o hasta caótica. Sin embargo, es necesario tomarle en cuenta, porque los hogares son espacios donde se realizan prácticas que se orientan al bienestar y la curación desde un conjunto de saberes y experiencias previas (William \& Popay, 1994, como se cita en Dew et al., 2104).

Comprender el hogar, y sus dinámicas en torno a la medicación, resulta imprescindible en el análisis de la autoatención y la automedicación, sobre todo si ambas son estudiadas como proceso y no necesariamente como un acto de consumo. En primer lugar, el hogar es el espacio donde se llevan a cabo las prácticas de medicación de forma descentralizada y sin supervisión del personal de salud; en segundo lugar, son espacios donde los bienes de consumo se apropian e individualizan donde una prescripción, un diagnóstico e inclusive un pronóstico, se combinan con los saberes de quien padece enfermedad y de miembros significativos; por tanto, los medicamentos (como bienes) son reconfigurados, apropiados o desechados; inclusive, dan por consecuencia el uso inapropiado de medicamentos y/o la falta de adhesión al tratamiento (Tilley, 2006, como se cita en Dew et al., 2014).

En tercer lugar, se profundiza en el hogar (en práctica de medicación) al posibilitar la producción de conocimiento acerca de la autoatención y automedicación desde varios dominios, científicos y no científicos, escindidos y distantes en apariencia, pero vinculados en la realidad. Este tipo de producción de conocimiento resulta importante, pues, al final, está relacionado tanto con el bienestar y la enfermedad, a la efectividad del tratamiento y a otros factores (Cárdenas, 1998; Dew et al., 2014).

\section{Autoatención doméstica de la salud, un bricolaje}

La autoatención doméstica de la salud es definida como una intervención que las personas hacen por cuenta propia en torno a la promoción y la prevención, la detección y el tratamiento; es un fenómeno que ocurre en el hogar. De forma habitual, comúnmente los líderes de hogar (en su mayoría adultos) desarrollan un primer diagnóstico ante la enfermedad o el padecimiento. Lo que hacen estos líderes o jefes de hogar puede ser visto como un bricolaje, como una serie de prácticas donde se adaptan los conocimientos dominantes (biomédicos) y los saberes y las experiencias previas de las personas (Cárdenas, 1998; Dew et al., 2014).

El bricolaje (bricoleur y bricolage) proviene de los conceptos utilizados por el antropólogo LeviStrauss, quien, inicialmente, buscó un abordaje donde el pensamiento "salvaje" y el pensamiento científico se explorarán desde su coexistencia, debido a que ambas formas de pensar intentan clasificar y sistematizar el pensamiento en relación con un fenómeno. En este caso, ambos tipos de pensamiento, científico y no científico son formas que permiten estructurar el mundo, tanto del profesional en salud, como del no profesional, sea este líder de hogar, o quien padece la enfermedad. En este caso el bricolaje, hace referencia, por un lado, al mundo mítico, a los significados, a las representaciones y las creencias populares que se practican en un espacio doméstico, es decir, en el hogar y que se viven cotidianamente; por otro lado, en el caso del pensamiento científico, usualmente es parte de lo expuesto desde la medicina y la farmacología.

Este concepto (bricolaje) expone que, tanto conocimiento científico como no científico carecen de hegemonía (uno sobre el otro), más bien, se propone que ambos pueden coexistir como un discurso 
AutOATENCIÓN Y AUTOMEDICACIÓN

híbrido, con emanaciones diferentes, pero con mecanismos similares, al menos, en el caso del estudio acerca de las prácticas domésticas (hogar) sobre la autoatención y la automedicación y su vivencia diaria (en y para lo cotidiano) (Levi-Strauss, 1968).

Pero, el estudio del bricolaje, en estos casos, no debe ser reducido al acto de consumo de medicamentos, sino que debe analizarse y estudiarse como un proceso dinámico, donde se entrelazan innumerables vínculos que son resultado de un sistema social de salud, de un sistema económico, político y social; que, a su vez, se entrelaza en un sistema cultural de salud, el cual se manifiesta en las prácticas del hogar - las cuales tienen por meta descentralizar y adaptar el conocimiento que viene del mundo científico, del espacio médico, y que se adaptan al hogar y a las posibilidades de quienes padecen la enfermedad(Cárdenas, 1998; Dew et al., 2014). Este análisis abre la posibilidad de estudiar a profundidad las dinámicas psicológicas, sociales y culturales que están subyacentes a la decisión del autoconsumo, de la autoprescripción, del autodiagnóstico, y claro, de la autoatención; todos estos son factores que pueden estar a la base de la ineficiencia e ineficacia de un tratamiento médico-farmacológico, de una falta de adhesión al tratamiento, del mal uso de medicamentos e inclusive, de una demanda baja en la consulta médica.

Muchas veces, a la base de estas decisiones, hay otros factores, como la percepción que se tiene de una enfermedad o padecimiento poco grave, la desconfianza y los prejuicios negativos acerca del sistema social de salud y el medicamento, la falta de tiempo y recursos económicos, el aumento gradual de las enfermedades en la población, lugares donde la enfermedad es considerada un estado normal, inclusive, o problemas en la relación entre profesionales en salud y usuarios (Cárdenas, 1998). En todo caso, el sistema doméstico de salud representa el primer nivel de asistencia, un nivel que ha sido poco estudiado y dimensionado desde una visión sistémica, donde este posee su propio entorno social, histórico, cultural, coyuntural y, no menos importante, político.

\section{Reflexiones finales}

El hogar es el primer nivel real de atención en salud, pues en dicho espacio se presentan acciones y manejos sociales donde emerge la autoatención. Este espacio (el hogar) también puede ser abordado como una categoría de investigación, pues se expresa una serie de "tácticas micro-subersivas", que suceden en la vida cotidiana; en este caso, fomentan y consolidan la autoatención como una práctica, como una acción de búsqueda y como una alternativa frente a las enfermedades, los padecimientos y los problemas que tradicionalmente son considerados afines a la salud y el bienestar.

Pero, esta práctica no solo debe ser comprendida de forma individual, pues forma parte en una estructura social, económica y política, es resultado y proceso y muchas veces se puede reflexionar desde la cadena de producción hasta la consolidación de la industria farmacéutica multinacional. Esta estructura impacta seres humanos, pues es por y para actores sociales y esto implica pensar al individuo no solo en términos individuales o endógenos, sino también en términos históricos, psicológicos, culturales, sociodemográficos y políticos.

El hogar presenta una serie de "prácticas laicas" (Dew et al., 2014) como se mencionó antes y, en estos espacios, emerge un bricolaje, que transforma y descentraliza el conocimiento científico desde el modelo biomédico y farmacológico. También, estas prácticas son resultado de una economía familiar, de creencias, prejuicios, significados y representaciones; es decir, implican un saber previo, un 
conocimiento que puede ser transmitido de generación en generación y este puede reflexionarse de forma multidisciplinar, pues su análisis a profundidad exige un pluralismo disciplinar y metodológico.

En la práctica, los espacios como el hogar y el cotidiano permiten comprender las jerarquías que algunos individuos hacen en la toma de decisiones respecto a su salud. Muchas veces, el estudio individual reportará que las personas asisten a consulta médica; sin embargo, a diario, la confianza no siempre está en el médico que diagnostica o prescribe, sino en otros actores sociales, como el farmaceuta, el botánico, el curandero, adultos en casa y ancianos; es decir, en una serie de recursos que forman parte de su estructura social, que se viven en comunidad y que forman parte medular en el estudio de la autoatención y sus partes, sean la automedicación, la autoprescripción o el autodiagnósto.

En consecuencia, pensar la autoatención implica una serie de retos, aparte de un abordaje multi disciplinar y claro, un pluralismo metodológico, implica ser pensada (la autoatención) como parte de un proceso y, a la vez, como el resultado de una serie de actores y agentes sociales, activos e inmersos en un sistema donde las prácticas médicas no son categorías puras, sino más bien categorías heterogéneas que exigen una reflexión que trasciende la farmacología y los hábitos de consumo o la biomedicina y la atención sanitaria en espacios profesionales.

Estas reflexiones, sobre el hogar y lo cotidiano, enmarcadas en una estructura y una totalidad social parten de la ontología del ser social y contribuyen no solo al campo de investigación y a la producción de conocimiento, sino también abonan a los esfuerzos de trabajo intersectorial en temas de salud y en el desarrollo de las comunidades. De nuevo, esto demanda retos, que no necesariamente son complicados, pero sí complejos, pues la reflexión desde el hogar y lo cotidiano evidencia la aún constante necesidad de un nuevo modelo de atención, orientado a la transformación del antiguo consultorio (Cárdenas, 1998). Esta reflexión lleva a las ciencias sociales y las ciencias de la salud a pensar más allá del cubículum, de lo vestal y de lo privado y en pensar en la autoatención y sus derivados como un proceso y no reducirle directamente a la trasgresión.

Es necesario enfatizar que los abordajes conceptuales y teóricos expuestos no deben ser entendidos como una pretensión de omnicomprensividad ante los fenómenos de autoatención y automedicación ni del hogar y la cotidianidad. La ontología del ser social y las concepciones afines permiten alcanzar un giro ontológico. No obstante, otras aproximaciones tienen el potencial de abordar de forma crítica los fenómenos mencionados. Un claro ejemplo son las perspectivas como la "crítica de la finitud" en Merrllasoux o "la teoría de ensamblaje y la doctrina de lo emergente" en Delanda. En ambos casos, es posible comprender cómo emerge el proceso de automedicación y autoatención y de qué manera es también parte de un entramado más amplio en la vida social, biológica y medio ambiental. Incluso, desde las teorías de los "entes escalares" en Escobar y Osterwell o desde el realismo crítico en Danemark representa una alternativa metodológica en la investigación social. Busca superar reduccionismos mediante el diálogo interdisciplinario y el análisis profundo de la naturaleza de la realidad. En todo caso, tienen el potencial de ampliar, complementar y dar respuesta a las limitaciones que el abordaje desde la ontología del ser social presenta (DeLanda, 2006; Danermark et al., 2016; Farias, 2008).

Desde las aproximaciones y las teorías psicológicas se comprende la automedicación desde diferentes perspectivas; por un lado, desde el abuso de sustancias como búsqueda para cambiar estados 
AutoATENCIÓN Y AutomedicACIÓN

internos que se consideran dolorosos; en este caso, se busca conocer los aspectos que abonan a la angustia subyascente que alimenta la adicción; por otro lado, como una forma de afrontar y buscar soluciones inmediatas, tanto para contrarrestar el dolor, como el padecimiento, incluso, si el primero es un dolor emocional (Hartney, 2021). En algunas ocasiones, la autoatención y la automedicación también surgen como forma de afrontar el dolor crónico, pues en ciertos casos los medicamentos recetados resultan deficientes. Otras posturas sostienen la idea de que la automedicación es una expresión de un comportamiento irresponsable o imprudente (Hartney, 2021).

Un reto será también pensar en un currículo y praxis para profesionales en salud, donde, aparte de tener una reflexión profunda y un debate en torno a la farmacovigilancia en sus campos profesionales, estos puedan otorgar a la autoatención doméstica de la salud el estatus que merece, pues a fin de cuentas, esta atención que se desarrolla en el hogar y en el cotidiano, es un primer real de asistencia sanitaria, y se vincula no solo al recurso económico, sino al individuo en todas sus dimensiones, en este caso, como un ser social.

Pensar y actuar de forma multidisciplinar en los estudios cuantitativos y cualitativos de utilización de medicamentos, es decir, de farmacocinética social, sin jerarquías canónicas y tradicionales demanda un reto y una reflexión necesaria, pues este pluralismo resulta indispensable en la comprensión de significados psicológicos, sociales y culturales tanto de la automedicación como de la autoatención y todas sus expresiones y variantes.

Emergen otros retos y reflexiones necesarias no solo en torno a los saberes, o al trabajo multidisciplinar y el pensamiento híbrido, sino también, en torno a pensar la interculturalidad y los derechos humanos en temas de autoatención y sus derivados. Pero, antes que nada, estos retos requieren una empatía genuina y menos discursiva o de panfleto y reclaman profundas reflexiones en torno a la sensibilidad y el respeto y, no menos importante, un ejercicio de humildad.

\section{Dedicatoria}

A mis profesores y colegas: Carlos Iván Orellana, Carlos Lara Martínez, Mario Zetino Duarte, Rafael Lara-Martínez, Marlon Carranza Zelaya, Esmeralda Valdivieso Mora, María Santacruz Giralt, Humberto Flores Muñoz, Amparo Cabrera González, Mike Anastario, Marilyn Deleón Castro, Federico Alegría Gómez, Meraris López Díaz, Flavio Menjívar Cartagena y Hugo de Burgos †. ¡ Gracias por creer en mí y fomentar la necesidad de incursionar en los fenómenos desde un pensamiento ontológico!

\section{Referencias}

Augé, M. (1995). Non-places. Introduction to an Anthropology of Supermodernity. Verso.

Ansari, M. (2018). Sociobehavioral Aspects of Medicines Use in Developing Countries. En M. I. Ibrahim, A. Wertheimer y Zaheer-Ud-Din Babar (Eds.), Social and Administrative Aspects of Pharmacy in Low-and Middle-Income Countries (pp. 15-33). https://doi.org/10.1016/B978-0-12-811228-1.00002-9

Brooks, S., Webster, R., Smith, L., Woodland, L., Wessely, S., Greenberg, N., \& Rubin, G. (2020). The psychological impact of quarantine and how to reduce it: rapid review of the evidence. Lancet, 395, 912-920. https://doi. org/10.1016/S0140-6736(20)30460-8 
Campos-Navarro, R. (2016). Antropología médica e interculturalidad. Sección VI Modelo médico de autoatención y modelo alternativo subordinado. McGraw Hill Education.

Cárdenas, E. (1998). Autoatención Doméstica de la Salud [Ponencia]. III Congreso Chileno de Antropología. Colegio de Antropólogos de Chile A. G., Temuco

Calderón, C., Soler, F., \& Pérez-Acosta, A. (2020). El Observatorio del Comportamiento de Automedicación de la Universidad del Rosario y su rol en la pandemia de COVID-19. Revista Ciencias de la Salud, 18(2), 1-8. http://www.scielo.org.co/scielo.php?script=sci_arttext\&pid=S1692-72732020000200001\&lng=en\&tlng= es

Castro, A., \& Farmer, P. (2003). El Sida y la violencia estructural: la culpabilización de la víctima. Cuadernos de Antropología Social, (17), 29-47.

Chicas, K. (2020, 25 de mayo). Pacientes que sufren de lupus aseguran no encontrar medicamento en farmacias para tratar la enfermedad. La Prensa Gráfica. https://www.laprensagrafica.com/salud/Pacientes-que-sufrende-lupusaseguran-no-encontrar-medicamento-en-farmacias-para-tratar-la-enfermedad-20200525-0043. $\underline{\mathrm{html}}$

Codina, L. (2020). Cómo hacer revisiones bibliográficas tradicionales o sistemáticas utilizando bases de datos académicas. Revista ORL, 11(2), 139-153. https://doi.org/10.14201/orl.22977

DeLanda, M. (2006). A New Philosophy of Society. Assemblage Theory and Social Complexity. Continuum.

Del Toro, M., Bohórquez, C., Díaz, A., \& Barrios, Z. (2020). Factors Associated with Self-Medication in Adolescents in the Rural area of Cartagena, Colombia. Archivos de Medicina, 20(2), 356-363. https://pesquisa.bvsalud. org/portal/resource/es/biblio-1118866

Danermark, B., Ekstrom, M., Jakobsen, L., \& Karlsson, J. (2016). Explicando la sociedad: el realismo crítico en las ciencias sociales. UCA Editores.

Dew, K., Chamberlain, K., Hodgetts, D., Norris, P., Radley, A., \& Gabe, J. (2014). Home as a hybrid centre of medication practice. Sociology of health \& illness, 36(1), 28-43. https://doi.org/10.1111/1467-9566.12041

Escobar-Salinas, J., \& Ríos-González, C. (2017). Automedicación en adultos de 11 ciudades de Latinoamérica, 2015-2016: estudio multicéntrico. Ciencia e Investigación Médico Estudiantil Latinoamericana CIMEL 22(2), 19-23. https://www.cimel.felsocem.net/index.php/CIMEL/article/view/706

European Environmental Bureau (2018, 24 de julio). The problem of pharmaceutical pollution. Autor. https://bit. ly/3kEwvE4

Fajardo-Zapata, A., Méndez-Casallas, F., Hernández-Niño, J., Molina, L., Tarazona, A., Nossa, C., Tejeiro, J., \& Ramírez, N. (2013). La automedicación de antibióticos: un problema de salud pública. Revista Salud Uninorte, 29(2), 226-235.

Farías, I. (2008). Hacia una nueva ontología de lo social Manuel DeLanda en entrevista. Persona y sociedad, 23(1), 75-85. http://redaprenderycambiar.com.ar/wp-content/lecturas/sociabilidad/DeLanda.PDF

Farmer, P. (2000). Desigualdades sociales y enfermedades infecciosas emergentes. Papeles de Población, 6(23), 181-201. http://www.scielo.org.mx/scielo.php?script=sci arttext\&pid=S1405-74252000000100009 


\section{AutoATENCIÓN Y AutOMEDICACIÓN}

Fernández, D., Pérez, Z., Cuevas, O., Quirós, M., Barrios, B., \& Dueñas, Y. (2021). Utilización de antibióticos en una población del municipio Cienfuegos. Medisur 19(1), 54-62. https://pesquisa.bvsalud.org/portal/ resource/es/biblio-1180830

Galindo, J. (2014). Erving Goffman y el orden de la interacción. Acta Sociológica, (66), 11-34. https://doi. org/10.1016/j.acso.2014.11.002

Gómez,C.,León,C.,\&Troya,A.(2020).Consumodepsicofármaco:unaproblemáticadesaludactual.MedicentElectrón 24(4), 826-832. http://scielo.sld.cu/scielo.php?script=sci_arttext\&pid=S1029-30432020000400826\&lng=e $\underline{\mathrm{s} \& \mathrm{tlng}=\mathrm{es}}$

González-López, J., Rodríguez-Gázquez, M., \& Lomas-Campos, M. (2012). Self-medication in adult Latin American immigrants in Seville. Acta Paulista de Enfermagem ACTA 25(2). https://www.scielo.br/j/ape/a/ BwXKMHRgSzTM4BVnzzm8r4M/?lang=pt

Hartney, E. (2021,26 de marzo). The Self-Medication Theory of Addiction. Verywell Mind. https://bit.ly/32a1P71

Heller, Á. (1977). Sociología de la vida cotidiana. Península.

Heller, Á. (2004). Lo cotidiano en la historia. Península.

Holmes, S. (2016). Fruta seca, cuerpos marchitos. Trabajadores agrícolas migrantes en Estados Unidos. AbyaYala.

Latour, B. (1993). We Have Never Been Modern. Harvard University Press.

Lévi-Strauss, C. (1968). Antropología Estructural. Instituto del Libro.

Lobo, H. H., \& Woranovicz, D. M. (2020). Consumer behavior towards pharmaceutical services: a scoping review. International Journal for Innovation Education and Research, 8(8), 326-340. https://doi.org/10.31686/ijier. $\underline{\text { vol8.iss } 8.2523}$

Manilowski, B. (1922). Argonauts of the Western Pacific: An Account of Native Enterprise and Adventure in the Archipelagoes of Melanesian New Guinea. Routledge \& Kegan Paul.

Márkus, G. (1974). Marxismo y antropología. Grijalbo.

Martínez-Cevallos, L. (2013). Percepción de la automedicación con antibióticos en los usuarios externos de un hospital público en Lima-Perú. Biblioteca virtual em saúde. http://pesquisa.bvsalud.org/portal/resource/pt/ $\underline{\text { lil-724574 }}$

Marx, K. (2005). Contribución a la crítica de la economía política. Introducción a la crítica de la economía política. Ediciones Cultura Popular.

Marx, K., \& Engels, F. (2007). La ideología alemana: crítica de la novísima filosofía alemana en las personas de sus representantes Feuerbach, B. Bauer y Stirner, y del socialismo alemán en las de sus diferentes profetas. Ediciones Cultura Popular.

Menéndez, E. (2003). Modelos de atención de los padecimientos: de exclusiones teóricas y articulaciones prácticas. Ciencia \& Saúde Coletiva, 8(1), 185-207. 
Menéndez, E. (2018). Autoatención de los padecimientos y algunos imaginarios antropológicos. Desacatos, 58, 104-113.

Ministerio de Salud y Bienestar Social de El Salvador. (2019). Atenciones a personas con lupus eritematoso sistémico, brindadas en los establecimientos del MINSAL, por diagnóstico, grupo de edades y sexo. Años 2016-2019. Dirección de Vigilancia Sanitaria de la Unidad de Estadística e Información en Salud.

Molina, J. (2020). S14 Lupus erythematosus and sleep hygiene: A salutogenic perspective. BMC Proceedings, 14 (22), 11. https://bmcproc.biomedcentral.com/articles/10.1186/s12919-020-00205-0

Molina, J., \& López, M. (2020). La salud mental en El Salvador: los costos invisibles de un problema olvidado. Un abordaje desde las Ciencias Sociales. En G. Burgos (Ed.) y Departamento de Economía UCA, Análisis socioeconómico de El Salvador (pp. 73-88). https://bit.ly/36iIQXv

Organismo Salvadoreño de Reglamentación Técnica (2016). Productos Farmacéuticos. Medicamentos Para Uso Humano. Farmacovigilancia. Reglamento Técnico. https://members.wto.org/crnattachments/2016/SPS/ SLV/16_3447_00_s.pdf

Organización Mundial de la Salud. (2020). COVID-19: cronología de la actuación de la OMS. Organización Mundial de la Salud. https://www.who.int/es/news-room/detail/27-04-2020-who-timeline-covid-19

Oviedo, H., Cortina, C., Osorio, J., \& Romero, S. (2021). Realidades de la práctica de la automedicación en estudiantes de la Universidad de Magdalena. Enfermería Global, 20(2), 531-544. https://doi.org/10.6018/ eglobal.430191

Pari-Olarte, J., Cuba-García, P., Almeida-Galindo, J., Aliaga-Guillén, N., Solano-García, C., Chacaltana-Ramos, L., Quispe-Ilanzo, M., \& Oyola-García, A. (2021). Factores asociados con la automedicación no responsable en el Perú. Revista del Cuerpo Médico del HNAAA CM 14(1), 29-34. https://dx.doi.org/10.35434/ remhnaaa.2021.141.867

Pardo, M. (1984). Patrones de automedicación. Cuadernos de Antropología. Antropología y Salud (3), 73-82.

Rajan, K. (2017). Pharmocracy. Value, Politics, and Knowledge in Global Biomedicine. Duke Press.

Sánchez, C., \& Nava, M. (2012). Análisis de la automedicación como problema de salud. Enfermería Neurológica 11(3), 159-162.

Vera-Romero, O., \& Vega-Romero, F. (2013). Diseño y validación de un Cuestionario para medir automedicación (CauM - ovr) en estudiantes universitarios. Cuerpo Médico, HNAAA 6(1), 19-24. 


\section{Sobre el autor}

Jorge Molina Aguilar es psicólogo, máster en Salud Mental, posee un posgrado en Psicooncología y cursa un Doctorado en Ciencias Sociales en el programa cotitulado de la Universidad Centroamericana “José Simeón Cañas" y la Universidad Don Bosco, El Salvador. Funge como miembro del "European Institute for Multidisciplinary Studies on Human Rights and Science" Knowmad Institut, Alemania. Escribe desde las Ciencias Sociales acerca de enfermedades crónicas, degenerativas y de lento progreso, el duelo y los comportamientos mortuorios. Ha presentado en congresos nacionales e internacionales a través de la Asociación Americana de Antropología Médica, la Asociación de Antropología Psicológica del Reino Unido y el Congreso Latinoamericano de Antropología. Forma parte como miembro de las divisiones 28 y 52 de la American Psychological Association, Psychopharmacology and Substance Abuse e International Psychology.

Publicado en línea: 28 de diciembre de 2021 